\title{
UNITS DURING HABITUATION, DISCRIMINATION LEARNING, AND EXTINCTION 1
}

\author{
T. Hirano ${ }^{2}$, P. Best ${ }^{3}$ and J. Olds ${ }^{4}$ \\ Brain Research Laboratory, University of Michigan, Ann Arbor, Mich. 48104 (U.S.A.)
}

(Accepied for publication: May 30, 1969)

Several experiment: (Galambos et al. 1956; Sharpless and Jasper 1956; Hernández-Peón 1960) showed that evoked potentials and arousal responses were often attenuated during habituation procedures. In some of these tests, habituated responses were later restored during conditioning. In our previous studies (Olds and Hirano 1969) unit responses were observed during alimentary conditioning after habituation trials. The conditioning caused hippocampal and midbrain units to become more responsive to the conditioned stimulus. It remained to be determined whether the augmented responses represented a return to the pre-habituated state, that is, whether the responses to conditioned stimuli were the same as responses to novel stimuli. Other studies apparently indicated that while novel stimuli and conditioned stimuli might have similar effects on the midbrain reticular formation, they probably had different effects on the hippocampus (Grastyán 1959; Adey et al. 1960; Pickenhain and Klingberg 1967).

In the present study, long-run recordings were made of neuron responses in several limbic and midbrain structures during habituation, condi-

1 This work was supported by research grants of the National Institute of Mental Health and the U.S. Public Health Service to J. Olds. The first author was Research Associate paid from the National Institute of Mental Health grants and the second author was a post-doctoral trainee with a stipend from the Physiological Psychology Training Grant from the National Institute of General Medical Sciences, U.S. Public Health Service.

2 Present address: Osaka City University, Department of Psychology, Osaka, Japan.

3 Present address: University of Virginia, Department of Psychology, Charlottesville, Va. 22901.

4 Present address: California Institute of Technology, Division of Biology, Pasadena, Calif. 91109. tioning and extinction. The primary aim was to find whether the responses that appeared in conditioning would ve the same or different from the ones that disappeared during the pseudoconditioning and habituation period. A secondary aim was to find whether the post-extinction state would be similar to that which was obtained after habituation but before conditioning.

To accentuate the possibility of observing differences between the pre-habituation responses, and to control for general changes in unit activity in individual cases, a discrimination procedure was used in which one auditory stimulus was a reinforced conditioned stimulus $(\mathrm{CS}+)$ and a different one was a non-reinforced "control" stimulus (CS-).

\section{METHODS}

The subjects were fifteen rats. Each was prepared with 6-8 indwelling micro-electrodes and a reference probe as described previously (Olds and Hirano 1969; Olds et al. 1969). Nichrome wires of $62.5 \mu$, enamel insulated at the factory and cut with scissors, were used as micro-electrodes without further preparation. These yielded unit responses of about $300 \mu \mathrm{V}$ amplitude, 500 $\mu$ sec duration, and 3:1 signal to noise ratio which were "found" or "lost" during initial surgery by probe movements of about $30 \mu$. Because of their constant amplitude and wave form and their dependence on probe location, these were considered to be the responses of individual neurons.

Recordings were made simultaneously from four probes during 2 sec intervals which included a 1 sec pre-stimulus interval and a 1 sec interval of auditory stimulation. A set of low-noise

Electroenceph. clin. Neurophysiol., 1970, 28: 127-135 
"microdot" cables was used to connect the animal via commutator and counterbalanced arm to the amplification system.

Movement of the animals was detected automatically. For this purpose one high-noise wire, open circuited at its lower end, was tied into the set of microdot cables. Its amplified signal provided a reliable index of movements by the animal. Animals. were required to remain "motionless" during data-taking procedures. That is, data were not accepted for computation if signals above a low background level were detected on the "movement" channel during the 2 sec datataking intervals. During conditioning proper, movements during the CS + caused not only rejection of the data but also cancellation of the reward.

Two auditory stimuli were used, 600 and 1000 c/sec tones, respectively, applied for a full second. One was the CS+ and the other the CS-. This was alternated from rat to rat. The unconditioned stimulus (US) was a $45 \mathrm{mg}$ food pellet supplied by a noisy food magazine with which the animals were familiar.

The two tones were presented repeatedly in a randomly interdigitated series until each tone had occurred in 650 movement-free trials. The first 300 of these were "pseudo-conditioning" trials. The second 200 were "conditioning" trials. The last 150 were "extinction" trials. The experiment was automatically programmed and continued day and night without interruption for a period of about 7 days. The presentation of the tones was randomized. On the average, one presentation of each tone was tried every $4 \mathrm{~min}$. But movement caused cancellation of about $75 \%$ of the trials and therefore a successful trial with each tone was made about once every $15 \mathrm{~min}$.

During the first 300 trials, habituation and pseudo-conditioning procedures were simultaneously employed. That is, in addition to the two tones presented on the schedule described above, pellets were discharged from the food magazine on a random schedule with an average of one every $4 \mathrm{~min}$. Recordings were made prior to and during the auditory stimuli. Movement prior to or during a tone caused its cancellation or termination and in either case the data were not counted. Movement during this period, however, did not affect the food reinforcement.
During the following 200 trials, the conditions were the same in all respects except that the US was presented not on a schedule of its own but after the completion of each 1 sec CS + application provided there was no movement during the 1 sec CS + interval. To assure attention to the US, animals were maintained on a limited diet for several days prior to commencement of the series of experimental tests so that body weight at the commencement of the series was at $80 \%$ of the level at the time of operation. Then during the 6-8 days of testing the animals received all of their food from the magazine. For this reason virtually all of the pellets presented by the foodmagazine were consumed immediately. During conditioning proper, the animals had to stand still during and just prior to the $\mathrm{CS}+$ in order to be fed. Because data were computed only for movement-free trials (during this and other parts of the experiment), it might be argued that all the data were obtained from successful trials. However, the animals had no way of anticipating the CS + (and therefore could not purposefully remain motionless prior to its presentation) and furthermore, the animals appeared to move or remain motionless largely by accident during $\mathrm{CS}+$ presentations even during the later trials of the conditioning series. This suggested that the capacity to become or remain motionless in response to a startling or meaningful stimulus was not in the operant repertory of these animals or was not acquirable in the limited time allowed.

During the final 150 trial "extinction" series, the habituation and pseudo-conditioning procedures were re-instituted, and conditions were exactly the same as in the pre-conditioning experiment.

Neuronal spikes were discriminated and counted automatically by a solid state device which took account of amplitude (by means of a window detector which set maximum and minimum limits) and spike duration (by means of a similar "time window"). This device has been described previously (Olds 1965).

After completion of the extinction series, the brains were sectioned and units were classified on the basis of probe location as determined from histological material. The $62.5 \mu$ probes left tracks which were clearly visible. Hippocampal probes were regularly in the ridge of pyramidal 


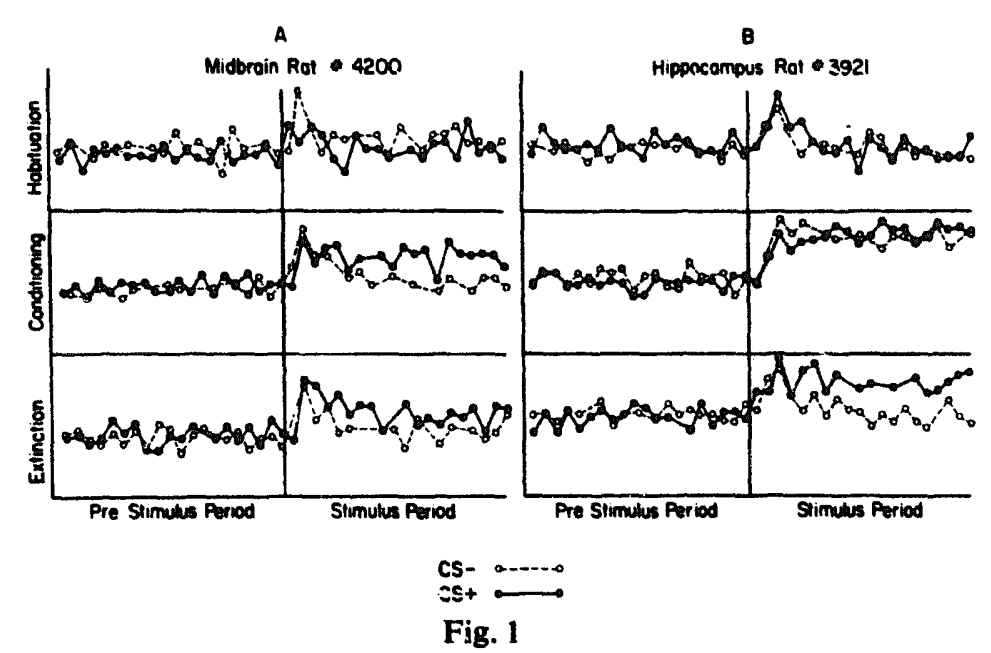

Average response curves for the habituation series, the conditioning series, and the extinction series for a hippocampal and a midbrain unit. The abscissa represents a 2 sec period without movement; it is divided into 50 msec intervals; the auditory stimulus was presented in the middle of the $\mathbf{2} \mathrm{sec}$ period and continued to the end. The ordinate represents neuronal firing rate in arbitrary units. In the following figures, the data of the second half are plotted as percentages of the mean value for the first half, thus indexing the response to the auditory stimulus in terins of the change it produced from the base rates for the first second.

cells in dorsal anterior hippocampus about evenly divided between the CA-1 and CA-3 fields. Midbrain probes were regularly in the area below the tectum, lateral to the central grey, and medial to the medial geniculate. Preoptic and hypothalamic probes were regularly in the lateral parts of these two areas.

\section{RESULTS}

In all areas there were increments in neuronal firing caused by the application of the auditory stimuli. In the midbrain these increments usually reached a maximum during the second $50 \mathrm{msec}$ interval after the stimulus onset (Fig. $1, A$ ). In hippocampus the maximum firing rate usually occurred during the third $50 \mathrm{msec}$ interval (Fig. $1, B)$. Before conditioning, the changes in rates of firing usually subsided by the end of 200-300 msec. They were therefore called "on-responses" and indexed by computation of the rate of action potentials during the first $\mathbf{3 0 0} \mathrm{msec}$ period as a percentage of the rate during the pre-stimulus control period $(1 \mathrm{sec})$. During the last $600-700$ msec of the $1 \mathrm{sec}$ stimulus period, the rate had stabilized in one of three states: (1) a sustained but greatly attenuated supra-normal firing rate, (2) a return to baseline, or (3) a sub-normal firing rate. Because rates were relatively constant during the last 600-700 msec, they were called "stabilized responses". An index of these was computed on the basis of the rate during the last $300 \mathrm{msec}$ period of the $1 \mathrm{sec}$ stimulus presentation period, expressing it as a percentage of the rate of firing during the pre-stimulus control period.

Because the patterns of discharge of the different units in a given brain area were similar to one another at a given stage in the experiment, not only the individual means for particular units were computed, but also the mean of these individual means for all units in an area (Fig. 2). One such grand average was computed for each each 25 trials in the course of the experiment and for each brain area. In each case, the 25 successive trials were averaged for each unit, and then the srores for the different neurons were averaged to derive the scores for a given brain area. Because one such score was obtained for each 25 trials, there were 12 such scores for the 300 habituation trials, 8 scores for the 200 conditioning trials, and 6 scores for the 150 extinction trials.

\section{Midbrain "on-responses"}

This is the rate of firing of neurons during the first 300 msec of the $1 \mathrm{sec}$ stimulus presentation

Electroenceph. clin. Neurophysiol., 1970, 28: 127-135 


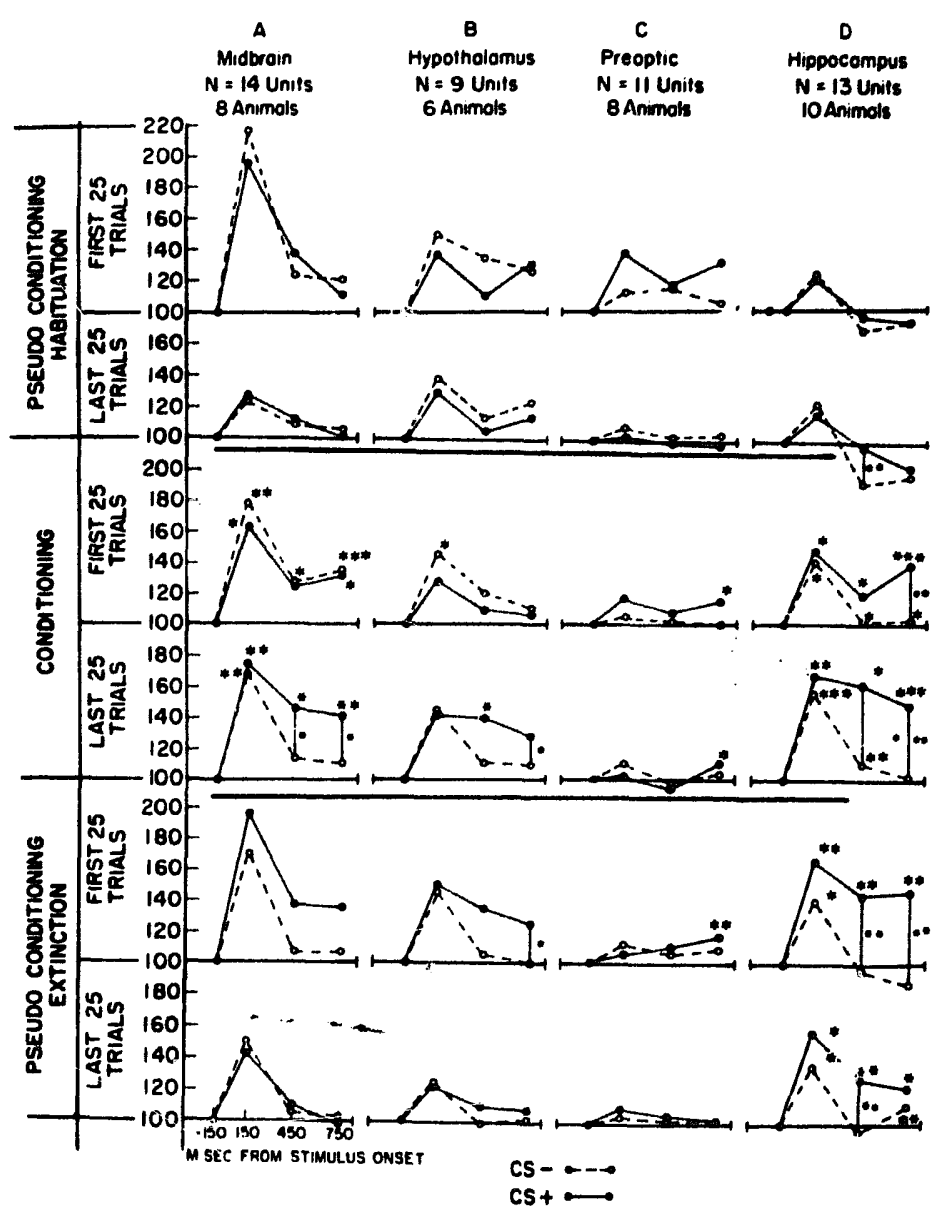

Fig. 2

Percentage changes in spike rates caused by $\mathrm{CS}-$ and $\mathrm{CS}+$ in early and late periods of pseudo-conditioning, conditioning and extinction. In each 4-point plot, the first point indicates the rate before stimulation $(100 \%)$ and the next 3 points indicate the rate for successive $1 / 3$ sec periods of stimulation as percentages of this base rate. Each curve represents the average of 25 trials for all units in a brain area. Although only data for the first and last 25 trials are presented, such curves were prepared for all 25 -trial intervals. From these prepared curves, points were extracted for Fig. 3 where the data for the onresponses are taken from the second point of the above plots, and the data for the stabilized-responses are taken from the fourth points (i.e. from the first and last thirds of the stimulus intervals). The ordinate is in percentage of pre-stimulus interval, and the abscissa is marked in msec by the midpoint of the successive 1/3 sec intervals. Asterisks indicate significance levels (Wilcoxon paired comparison test) as follows: *, 0.05 level; ${ }^{* *}, 0.01$ level; ***, 0.001 level. The large asterisks above the CS+ and CSlines indicate differences from the habituation level (established by the last 25 trials of pseudo-conditioning habituation); the small asterisks appended to vertical lines between the two curves indicate differences between the CS+ and CS - responses.

period. The "on-responses" to the CS + and the CS- declired steadily during the pseudo-conditioning, habituation series of trials. This "onresponse" started at a height of $200 \%$ of control during the first 25 habituation trials and fell to $130 \%$ during trials $275-300$ (Fig. $3, A$ ). The rate of firing rose back to $170 \%$ during the first 25 conditioning trials and remained at this level throughout the remainder of these trials (Fig. 4, $A$ and $B$ ). While there was no difference between the midbrain "on-response" to the CS+ and CS - during the conditioning trials, during the first 75 trials of extinction a difference appeared: "on-responses" to the CS + rose to $200 \%$ during the first $\mathbf{5 0}$ trials of extinction, whereas the "on-responses" to the CS - returned to the 


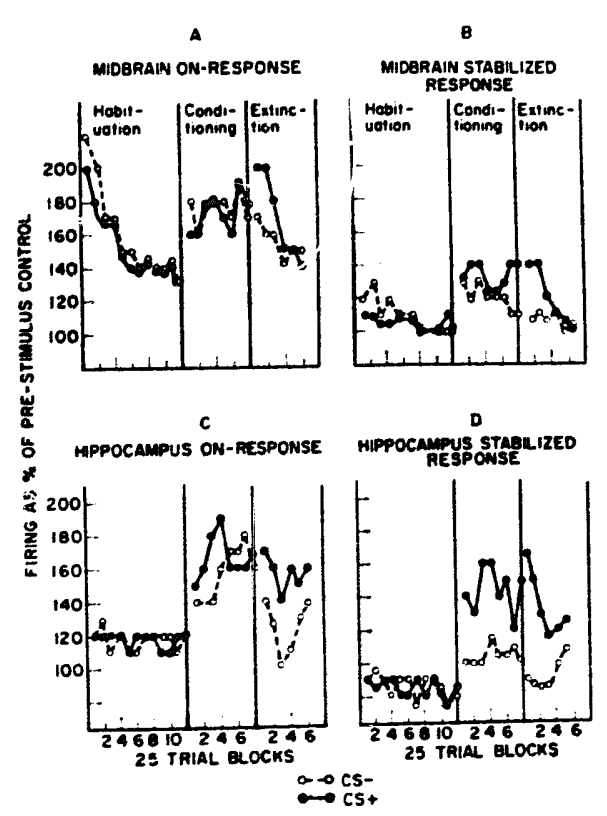

Fig. 3

The course of habituation, conditioning and extinction in 25-trial blocks for the on-rosponse and the stabilizedresponse for the midbrain and the hippocampus. The data are taken from grand average plots as shown in Fig. 2.

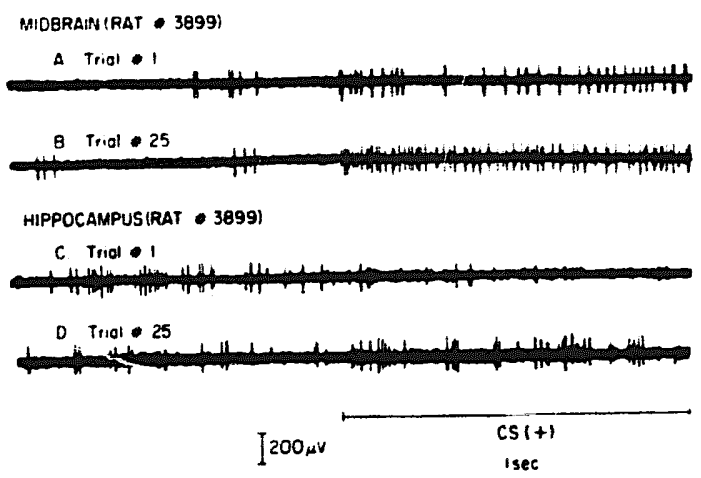

Fig. 4

Neuronal firing from the midbrain and hippocampus during application of the CS + before and after 25 trials of the conditioning experiment. Note the habituated state of the midbrain unit during trial 1 as compared with the conditioned state during trial 25 . Note also the suppression of the hippocampal unit during the CS + prior to conditioning and the acceleration during the same period after conditioning.

pre-conditioning levels. During the last 75 extinction trials this difference vanished, and the mean "on-response" to CS+ and CS - was $140 \%$, not significantly above the pre-conditioning level.
Midbrain "stahlized responses"

This is the rate of firing of neurons during the last $300 \mathrm{msec}$ of the $1 \mathrm{sec}$ stimulus presentation period. The "stabilized responses" in midbrain were very small $(115 \%)$ at the beginning of habituation trials (Fig. 3, B). From this initial low rate of discharge, there was a decline to control levels as habituation proseeded. These "stabilized responses" then rose to $140 \%$ during the initial conditioning trials and remained at this level during the whole series of such trials. They fell to control levels again during extinction as they had during habituation. Late in the series of conditioning trials, a large difference between the "stabilized responses" to the CS + and CS appeared. It was statistically significant only after 150 conditioning trials. From then on, the "stabilized responses" to the CS - were reduced toward control levels even during continued conditioning trials, while the "stabilized responses" to the CS+ remained at $140 \%$. This difference in rate of discharge of midbrain neurons in response to CS + and CS - lasted during the first 75 extinction trials. Then during the remainder of the extinction trials, the "stabilized responses" to the CS + and CS - were again similar and at or near the zero level.

\section{Hippocampus "on-responses"}

No change in the "on-responses" to either the $\mathrm{CS}+$ or CS- occurred from block to block during the course of the habituation (pseudoconditioning) trials (Fig. 3, C). Because the habituation data were analyzed by compute: in 25-trial blocks, it was not possible to observe changes that might have occurred within the first block as a result of a very small number of habituation trials, but there were clearly no changes from block to block. The "on-responses" were $120 \%$ of control during the first 25 habituation trials and during trials 275-300 which were the last 25 trials of this series. During the first 25 conditioning trials, however, the "onresponses" to the $\mathrm{CS}+$ rose to $150 \%$ of control rates of firing (Fig. 4, C and $D$ ) and the "or:responses" to the CS - rose to $140 \%$ of control values. During the next 75 conditioning trials, the CS + "on-responses" rose to a maximum of $190 \%$. The "on-responses" to the CS - rose more slowly and there was a significant difference

Electroenceph. clin. Neurophysiol., 1970, 28: 127-135 
between the two responses throughout condiiioning trials 25-75. During the last 100 conditioning trials, these "on-responses" to both CS+ and CS - levelled off at about $165 \%$ of control firing rates and the difference between the firing rates induced by the two kinds of stimuli disappeared. During extinction trials, the "on-responses" to the CS + were reduced slightly from 165 to $155 \%$, while the "on-responses" to the CS fell to about $135 \%$. The difference between the two "on-responses" thus reappeared in extinction; it was significant during four of the six 25trial blocks. The "on-responses" at the end of extinction were significantly above those at the end of habituation (Fig. 3, C).

\section{Hippocampus "stabilized responses"}

During habituation trials, these "stabilized responses" remained consistently at about $80-90 \%$ of spontaneous firing rates. During coriditioning trials the "stabilized responses" to the CS + increased abruptly from their sub-normal values of $80-90 \%$ to supra-normal values of about $140 \%$ of spontaneous firing rates. This occurred in the course of the first 25 trials, and the "stabilized responses" remained at about this level during the remainder of the conditioning trials (Fig.3, D). The "stabilized responses" to the CS - increased from the sub-normal $(80-90 \%)$ levels up to levels of about $100 \%$; in other words, the subno."nal "stabilized responses" dis.appeared but they were not replaced in this case by any significant supra-normal "stabilized responses". There was a significant difference between the "stabilized responses" induced by CS + and CS - during each one of the eight blocks ( 25 trials each) of the conditioning series of trials. During extinction, the "stabilized responses" to the CS + declined to $120 \%$ of spontaneous firing rates during the first 75 extinction trials and then remained at this level (30-40 percentage points above the preconditioning level) throughout the remainder of the series of extinction trials. The "stabilized responses" to the CS - levelled off at the spontaneous firing rate $(100 \%)$ significantly below the "stabilized response" to the CS + (120\%).

\section{Hypothalamic "on-responses" and "stabilized responses" (Fig. 2, B)}

The "on-responses" amounted to $140 \%$ of pre-stimulus, spontaneous firing during most of the three different series of trials, i.e., there was no appreciable change during habituation or conditioning trials. Furthermore, no difference between the "on-responses"'induced by CS+ and CS - were observed. During the early extinction trials the "on-responses" to both stimuli rose moderately (to $155 \%$ ) and then declined (to $120 \%$ ) in the further course of extinction trials. The hypothalamic "stabilized responses" declined from 130 to $110 \%$ during habituation trials. During conditioning trials, the "stabilized responses" to the CS + increased gradually to the $130 \%$ level, whereas the "stabilized responses" to the $\mathrm{CS}$ - remained at the pre-conditioning values.

Preoptic "on-responses" and "stabilized responses" (Fig. 2, C)

Preoptic neurons were relatively unmodified by auditory stimulation. The "on-responses" declined from 125 to $105 \%$ of spontaneous firing rates during habituation, but the change was not statistically significant. The "on-responses" to $\mathrm{CS}+$ and $\mathrm{CS}$ - then remained at a level of $110 \%$ during subsequent conditioning and extinction trials. The preoptic "stabilized responses" showed the same pattern of changes as that of the "onresponises."

\section{DISCUSSION}

A substantial change between the first and last 25-trial blocks in the initial pseudo-conditioning series was interpreted as indicating that a response underwent habituation. $A$ large increase above the pre-conditioning level during conditioning trials was assumed to indicate that a response was modifict by conditioning. A subsequent decline to pre-conditioning levels was evidence of its extinction. Any large differences between $\mathrm{CS}+$ and $\mathrm{CS}$ - responses during conditioning or extinction provided evidence that the response exhibited discrimination.

The midbrain "on-responses" exhibited habituation, conditioning, and extinction. There was discrimination only during the early part of the extinction series and this was not statistically reliable. The midbrain: "stabilized responses" also underwent habituation, conditioning and 
extinction. In this case there was clear discrimination exhibited during conditioning. It did not appear until late in the conditioning series and it was statistically significant only during the last two blocks of conditioning (trials 150-200).

The hippocampal "on-responses" exhibited conditioning. There was no prior habituation because a large response in this area did not appear until after conditioning. Surprisingly there was no indication that extinction was complete or still underway at the end of the 150-trial extinction series; the response declined at first but then stabilized and gave no hint of further decline during the last 75 extinction trials. In the end it was far above and statistically above the preconditioning levels. Discrimination appeared and was statistically significant during the first part of the conditioning series (trials 25-75); it disappeared during the latter half of conditioning (trials 100-200) and then reappeared in the subsequent extinction series. The hippocampal "stabilized responses" exhibited all of the same features as the hippocampal "on-response" but all of the effects were larger and more significant. There was no change during habituation; the response started out below control levels and stayed there. There was marked conditioning which appeared immediately and lasted through all eight 25-trial blocks. There was no extinction nor any sign that extinction was continuing beyond the 100th extinction trial. Most remarkable was the total discrimination that appeared significantly during the first 25 conditioning trials and was then exhibited in all 25-trial blocks of conditioning and extinction.

The dramatic difference between the midbrain and hippocampal units should be noted. The midbrain units showed clear and continuous habituation during the long series of preliminary trials, and a similar course of changes during the final extinction trials. The hippocampal units showed no particular change between the first 25 trials of habituation and trials 275-300; and the "new" responses that appeared in the course of conditioning did not disappear in extinction. While there was some attenuation, this was complete by the end of the first 100 extinction trials, after which there was no further trend toward extinction of the still-significantly augmented hippocampal response. It seemed as if the hip- pocampal firing rates were set in one steady state all during the habituation procedure, and had been shifted to a different steady state by the end of the extinction series. Perhaps a further series of extinction trials or time alone would eventually have shifted the firing rate back to that which had been observed during habituation; but the last $\mathbf{5 0}$ extinction triais haci no discernible effect. Correlated with this difference between midbrain and hippocampus in regard to habituation and extinction was a difference in discrimination: discrimination appeared to be rapid and relatively stable in hippocampus and it did not extinguish; discrimination was slow in appearing in midbrain and it disappeared readily in extinction.

Caution is required in comparing the hippocampal data reported here with that of previously reported experiments (Grastyán 1959; Adey et al. 1960; Pickenhain and Klingberg 1967) for three reasons: First, most of the older data were obtained by observation of hippocampal slow wave activity while the present data were obtained by observation of unit responses. As it is not clear whether a negative or positive relation exists between the two, they must not be considered as correlated indexes of hippocampal activity. Second, most of the older reports involved a relatively small number of trials. In the present experiment, the large number of trials in each block may have permitted an early and rapid habituation process in hippocampus to occur without being observed. Previous reports of Grastyán (1959) and Pickenhain and Klingberg (1967) have indicated that startle reactions and correlated hippocampal desynchronization occurred only during the first few trials, and that thereafter, hippocampal slow waves occurred during orientation responses. This set of processes and even the later habituation of the orientation reactions and the associated hippocampal slow wave responses might have been completed during the first 25 trials in our experiment and it might therefore have escaped our cbservation. In fact, because our "conditioning" of hippocampal units was to all intents complete by the end of 25 trials, it seems particulary likely that habituation might have proceeded to completion with similar rapidity. Third, most of the older experiments involved overt and covert move- 
ments associated with orienting and startle reactions. The overt and even many of the covert movements would have caused our data-taking apparatus to reject the data and therefore the data in the present experiment were collected from trials during which such movements did not occur. Because it seems particularly likely that some hippocampal EEG changes, particularly the ones observed early in habituation, are associated with such movements, it should not be too surprising if these early changes did not appear in our data.

In our experiment, the midbrain units responded with some similarity to novel stimuli and conditioned stimuli, and failed to respond to habitudted and extinguished stimuli. The hippocampal units, on the contrary, failed to respond to novel stimuli or habituated stimuli, but responded to conditioned stimuli during conditioning and even after extinction. The failure of response to novel stimuli and the response to conditioned stimuli were superficially parallel to the findings of Grastyán (1959) and Pickenhain and Klingberg (1967) with observation of hippocampal slow waves. However, because of the difference in time course and dependent variables, there is no strong reason to believe that these were related processes. Grastyan supposed that the hippocampal slow wave response was the concomitant of the orientation response, and Pickenhain and Klingberg supposed that an information "comparator" in hippocampus served a mediating role in non-automatic, purposive behavior. The latter view was also consistent with the experiments of Adey et al. (1960). We have suggested (Olds and Hirano 1969) that hippocampus might serve as a temporary memory register, controlling automatic response to a stimulus on the basis of recent memories. While this is a concept similar to that of Pickenhain and Klingberg, it nevertheless involves the contrary assumption that during non-automated behavior, hippocampal slow waves appear representing a disengaged (non-operative) state of hippocampus while neocortex is in control. Later when the behavior becomes automated, the absence of slow waves indicates that hippocampus is reengaged and involved in controlling the automatic conditioned response on the basis of its relatively temporary mnemonic traces. Such a view is highly speculative; but it is not out of harmony with the present data. The trace in hippocampus would appear in the course of acquisition trials and would remain in control of behavior all through conditioning. After extinction, behavioral aspects of the trace might disappear, but mnemonic aspects might remain in the set of temporary traces until displaced or erased as a function of new material requiring storage space or, equally likely, by time alone.

\section{SUMMARY}

Unit responses were recorded with $62.5 \mu$ fine wires implanted chronically in rats. In each animal simultaneous recordings were made from four probes: in hippocampus, midbrain, hypothalamus and preoptic area. Hippocampal units did not respond in a special fashion to novel stimuli and what responses there were did not change during habituation. After this, response increments appeared rapidly in hippocampus during conditioning, and at the same time a difference developed between the hippocampal firing rates induced by the CS + and CS - control stimulus. In extinction, the responses in hippocampus changed again to a level between the habituation level and the conditioning level. In other words, complete extinction oi the increments in firing rates brought on by conditioning did not occur.

Midbrain units responded to novel stimuli initially, and then showed clear and continuous habituation during the preliminary series of trials. They showed increments in rate of discharge during conditioning; and a difference between patterns of firing induced by $\mathrm{CS}+$ and $\mathrm{CS}-$ developed gradually as compared to its rapid appearance in the hippocampus. The midbrain pattern of neuron responses which appeared during conditioning disappeared during extinction.

Hypothalamic units showed patterns of neuron responses somewhat similar to those of midbrain units but less pronounced.

Preoptic units showed relatively small responses to novel stimuli but these disappeared rapidly during habituation and there were no further responses in the series of conditioning or extinction tials.

Electroenceph. clin. Neurophysiol., 1970, 28: 127-135 
RÉSUMÉ

ACTIVITÉ UNITAIRE AU COURS DE L'HABITUATION, DE L'APPRENTISSAGE DISCRIMINATIF ET DE L'EXTINCTION

Les réponses unitaires ont été enregistrées avec des électrodes implantées chroniquement chez des rats, d'un diamètre de $62,5 \mu$. Chez chaque animal, des enregistrements simultanés ont été pratiqués à partir de 4 sondes: dans l'hippocampe, le mésencéphale, l'hypothalamus et l'aire pré-optique. Les unités hippocampiques ne répondent pas de façon particulière à des stimuli nouveaux et les réponses quelles qu'elles soient ne changent pas au cours de l'habituation. Ensuite, des accroissements de réponses apparaissent rapidement dans l'hippocampe au cours du conditionnement et en même temps on note une différence entre les taux de décharges hippocampiques induits par le stimulus conditionnel positif (C.S. +) et le stimulus conditionnel négatif (C.S. - ) de contrôle. Au cours de l'extinction, les réponses au niveau de l'hippocampe changent encore pour atteindre un niveau intermédiaire entre le niveau de l'habituation et le niveau du conditionnement. En d'autres termes, l'extinction complète de l'accroissement du taux de décharges élaboré par le conditionnement ne survient pas.

Les unités du mésencéphale répondent à des stimuli nouveaux initialernent, puis montrent une habituation nette et continue au cours des séries préliminaires de séquences. Elles montrent un accroissement de leur taux de décharges au cours du conditionnement; une différence entre les patterns de décharges induits par le C.S. + et le C.S. - se développe de façon progressive, comparée à son apparition brusque dans l'hippo. campe. Au niveau du mésencéphale, le pattern de réponses neuroniques qui apparaît au cours du conditionnement, disparaît pendant l'extinction.
Les unités hypothalamiques montrent des patterns de réponses neuroniques quelque peu similaires à celles des unités du mésencéphale, mais moins prononcés.

Les unités pré-optiques montrent cies réponses relativement petites à des stimuli nouveaux mais qui disparaissent rapidement au cours de l'habituation; aucune réponse ultérieure ne s'observe au cours des séquences de conditionnement ou d'extinction.

The authors are grateful to G. Baldrighi for preparation of many animals, to $\mathbf{W}$. Wetzel for construction and maintenance of the automated behavioral devices, to W. Allan for assistance in carrying out the experiment and to $\mathbf{J}$. Frey for histological preparations.

\section{REFERENCES}

Adey, W. R., Dunlop, C. W. and Hendrix, C. E. Hippocampal slow waves. Arch. Neurol. (Chic.), 1960, 3: 74-90.

Galámsos, R., Sheatz, G. and Vernier, V. G. Electrophysiological correlates of a conditioned response in cats. Science, 1956, 123: 376-377.

GrastYÁN, E. The hippocampus and higher nervous activity. In M. A. B. BrAzIER (Ed.), The central nervous system and behavior (Trans. 2nd Conf.). Macy Foundation, New York, 1959: 119-205.

Hernández-Peón, R. Neurophysiological correlates of habituation and other manifestations of plastic inhibition (internal inhibition). Electroenceph. clin. Neurophysiol., 1960, Suppl. 13: 101-114.

OLDs, J. Operant conditioning of single unit responses. Proc. XXIII int. Congr. physiol. Sci., Tokyo, 1965: 372-380.

Olds, J. and Hirano, T. Conditioned responses of hippocampal and other neurons. Electroenceph. clin. Neurophysiol., 669, 26: 159-166.

Ol.Ds, J., MiNK, W. D. and BEsT, P. J. Single unit patterns during anticipatory behavior. Electroenceph. clin. Neurophysiol., 1969, $26:$ 144-158.

Pickenhain, L. and Klingeerg, F. Hippocampal slow wave activity as a correlate of basic behavioral mechanisms in the rat. Progr. Brain Res., 1967, 27: 218-227.

Sharpless, S. and Jasper, H. H. Habituation of the arousal reaction. Brain, 1956, 79: 655-680.

Reference: HIRANO, T., BEST, P. and OLDs, J. Units during habituation, discrimination learning, and extinction. Electroenceph. clin. Neurophysiol., 1970, 28: 127-135. 